\title{
Introduction : Autorité et Web 2.0
}

Introduction: Authority and Web 2.0

\section{Stefano Vicari}

\section{(2) OpenEdition}

\section{Journals}

\section{Electronic version}

URL: http://journals.openedition.org/aad/4936

DOI: $10.4000 /$ aad.4936

ISSN: 1565-8961

\section{Publisher}

Université de Tel-Aviv

\section{Electronic reference}

Stefano Vicari, "Introduction : Autorité et Web 2.0", Argumentation et Analyse du Discours [Online], 26 | 2021, Online since 12 April 2021, connection on 16 April 2021. URL: http://journals.openedition.org/ aad/4936 ; DOI: https://doi.org/10.4000/aad.4936

This text was automatically generated on 16 April 2021.

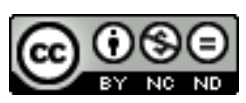

Argumentation \& analyse du discours est mis à disposition selon les termes de la licence Creative Commons Attribution - Pas d'Utilisation Commerciale - Pas de Modification 4.0 International. 


\title{
Introduction : Autorité et Web 2.0
}

\author{
Introduction: Authority and Web 2.0
}

\author{
Stefano Vicari
}

\section{Objectifs du numéro}

1 Depuis l'Antiquité, avec Platon, et jusqu'à nos jours, la notion d'autorité a fait l'objet de nombreuses réflexions sous la plume des philosophes, sociologues, psychologues. Cette réflexion s'est surtout développée à partir du $20^{\mathrm{e}}$ siècle, tantôt dans le but de la spécifier par rapport à des notions voisines avec lesquelles elle est souvent confondue, comme celles de pouvoir et de domination (Arendt 2004 [1955]), tantôt pour établir la typologie de ses grandes figures (Weber 1922, Kojève 2004), tantôt encore pour montrer le fondement symbolique, psychologique, idéologique de la soumission à l'autorité (Durkheim 1925, Adorno et al. 1950, Bourdieu 1975). Ses liens avec le mécanisme psychologique de l'obéissance (Milgram 1963) et avec la notion de vérité, dans sa version coercitive (Foucault 1975) ou libératrice (Foucault 2009) ont été également analysés. Des chercheurs ont exploré la dimension juridique de l'autorité (Raz 1979), ou ont insisté sur sa double nature socio-relationnelle et langagière, surtout à la suite de Bochenski (1974). Ils ont ainsi contribué à montrer le rôle du langage dans l'établissement de l'autorité. Enfin, plus récemment, épistémologues, sociologues et spécialistes en sciences de l'information et de la communication ont mis en lumière son caractère historicisé (Leclerc 1996) et les changements, voire les distorsions, provoqués par les liens que l'autorité entretient avec les phénomènes de popularité et de réputation sur les réseaux sociaux (Origgi 2008, Alloing 2014, Candel et GkouskouGiannakou 2017, Broudoux et Ihadjadene 2020).

2 L'autorité est une notion plurielle, dynamique, dont la nature multidimensionnelle fait un objet de recherche non seulement transversal à tous les champs des sciences humaines, mais aussi difficile à cerner et encore plus à définir. Néanmoins, l'accord sur sa double nature discursive et socio-relationnelle justifie amplement une étude de son fonctionnement et de sa circulation dans et par les discours, notamment ceux qui sont produits dans l'univers discursif numérique, hétéroclite et mouvant, du web 2.0, où il 
semblerait que la distinction nette entre locuteurs légitimes et " sans voix " de Bourdieu (1977) perd de sa pertinence.

3 L'objectif de ce numéro est de mieux comprendre quelles conceptions de l'autorité discursive ressortent de l'analyse de cas d'études concrets portant sur ce terrain si particulier qu'est le web 2.0. Les contributions permettent de montrer non seulement dans quelles conditions discursives et à l'aide de quelles stratégies les locuteurs construisent des "discours d'autorité », et accordent ou refusent leur confiance à une autorité ; mais aussi dans quelle mesure et de quelle manière cette notion se modifie dans ces espaces discursifs où les locuteurs ne partagent pas forcément les mêmes savoirs ni les mêmes représentations sur les questions traitées.

\section{Autorité, Analyse du discours et Argumentation}

\section{Aux origines : idéologie, formation discursive et archive}

La «question » de l'autorité est centrale pour l'Analyse du Discours (dorénavant AD), depuis ses origines. Le projet, élaboré dans la conjoncture intellectuelle de la fin des années 60 en France, dans le sillage des réflexions philosophiques d'Althusser et de Foucault, s'inscrit dans une démarche foncièrement critique de l'autorité politique via la déconstruction des représentations idéologiques dont le politique se fait le vecteur. La tâche de l'AD serait de " démystifier » l'idéologie, de donner une " lecture objective » de phénomènes lexico-syntaxiques propres au discours politique afin de comprendre les liens entre effets d'évidence et construction de l'autorité en discours (Oger 2019).

Définie comme "une représentation du rapport imaginaire des individus à leurs conditions réelles d'existence " (Althusser 2006 [1970] : 114), la notion d'idéologie y joue un rôle fondamental. Sa fonction d'interpellation non seulement lui confère une dimension analysable du point de vue linguistique, mais elle permet aussi de définir le statut du sujet par rapport à l'idéologie elle-même : « un être assujetti, soumis à une autorité supérieure, donc dénué de toute liberté, sauf d'accepter librement sa soumission » (ibid. : 140). C'est dans l'explication de l'assujettissement de l'individu à l'idéologie portée par les appareils idéologiques d'État qu'Althusser recourt au terme d'« autorité » pour référer à l'idéologie. Il s'agirait d'une instance "supérieure » qui limite la liberté de l'individu et qui tire son pouvoir du fait qu'elle agit au niveau imaginaire, de l'inconscient. Les appareils idéologiques et répressifs d'État contribueraient à la légitimation et à la perpétuation d'idéologies particulières qui les soutiennent, et à la reproduction du système des rapports de pouvoir entre les classes sociales.

6 C'est avec la publication de l'Archéologie du savoir (Foucault 2008 [1969]), qui contribuera à nourrir les réflexions des analystes du discours, que se pose la question de la légitimité des instances énonciatives (Maingueneau 1992): "qui parle? Qui, dans l'ensemble de tous les individus parlants, est fondé à tenir cette sorte de langage?» (Foucault 2008 [1969]: 72). Pour répondre à ces questions, Foucault insiste sur la nécessité de prendre en compte les modalités énonciatives propres à une formation discursive donnée, celle-ci étant «Un ensemble de règles anonymes, historiques, toujours déterminées dans le temps et l'espace qui ont défini une époque donnée, et pour une aire sociale, économique, géographique ou linguistique donnée, les conditions d'exercice de la fonction énonciative » (ibid. : 153). 
7 Le projet foucaldien prévoit donc l'historicisation des modes de production institutionnels, sociaux et politiques d'un discours de vérité qui tire son autorité du respect de règles et de techniques (Foucault 1975) de création du savoir suivant les différentes périodes culturelles, afin de faire ressortir les régularités régissant la diversité des objets discursifs. Ces régularités permettent aux textes d'accéder à l'archive, à savoir «le système général de la formation et de la transformation des énoncés » (ibid. : 177) qui détermine ce qui peut être dit à une époque donnée.

Reprise par Maingueneau (1992), cette notion permet d'élargir les domaines d'analyse à d'autres corpus que les corpus politiques, pour englober l'ensemble des "discours autorisés, qui au-delà de leur fonction immédiate supposent, à travers les renvois interdiscursifs, un rapport aux fondements et aux valeurs » (Maingueneau 1993: en ligne), où une place privilégiée est faite aux discours constituants (Maingueneau et Cossutta 1995). Ces discours, qui relèvent des domaines religieux, scientifique, philosophique, littéraire et juridique, posent avec force la question de comprendre quels phénomènes discursifs leur permettent de s'auto-légitimer par le seul fait d'être énoncés.

\section{La centralité de la question de l'autorité en AD entre légitimation et crédibilisation}

9 Sous la double influence de la notion d'archive reprise par Maingueneau en AD et de la théorie des champs de Bourdieu, et des recherches de M. de Certeau (1974), l'AD connaît ce que Maingueneau qualifie de «tour ethnolinguistique ». Celui-ci détermine la préférence des chercheurs pour l'analyse des fonctionnements de discours produits par des groupes plutôt homogènes et délimités par des champs suivant des règles spécifiques (Oger 2005).

10 La question de l'autorité apparaît en effet comme transversale à différentes approches qui ont animé l'AD depuis les années 80 jusqu'à nos jours et qui justifient l'entrée " autorité » dans le Dictionnaire d'Analyse du Discours (2002), la consacrant comme l'une des notions-clés de la discipline.

11 L'articulation de la problématique de l'autorité avec celle du fonctionnement du discours, se fait via les deux opérations discursives de légitimation et de crédibilisation. La légitimation renvoie à la "qualité de celui qui est fondé à agir comme il agit». La légitimité serait donc le « résultat d'une reconnaissance par d'autres de ce qui donne pouvoir de faire ou de dire à quelqu'un au nom d'un statut » (Charaudeau 2005 : 52). Elle se distingue de la crédibilisation en ce qu'elle « détermine un "droit du sujet à dire et à faire", alors que la seconde détermine "une capacité du sujet à dire et à faire" » (ibid.). La crédibilisation relève donc plutôt d'une " construction opérée par le sujet parlant de son identité discursive de telle sorte que les autres soient conduits à le juger digne de crédit » (ibid. : 91).

12 La parole légitimée serait le résultat de deux processus : une « autorité institutionnelle " fondée sur une autorité déontique, à laquelle est conféré un certain pouvoir de décision et/ou une autorité épistémique, régie sur un statut particulier du sujet (expert, etc.) et une " autorité personnelle ", qui tient à la capacité de persuasion du sujet. Ces opérations constituent les dynamiques permettant de circonscrire la question de l'autorité telle qu'elle a été développée en $\mathrm{AD}$ à partir des travaux sur les genres de 
discours, sur l'évidence de la doxa et du sens commun et, enfin, sur les problématiques énonciatives.

13 Les analystes du discours qui ont mené une réflexion sur les genres de discours (Maingueneau 2010, Rastier 2001, Beacco 1992) ont montré que le respect des régularités imposées par les genres institués permet de légitimer le discours du locuteur et autorise en quelque sorte sa prise de parole (Guilbert 2014). Cela est bien visible dans les recherches sur les genres institutionnels (Oger et Ollivier-Yaniv 2003) ainsi que dans les études qui portent sur les genres professionnels, littéraires, philosophiques ou médiatiques où la légitimité du locuteur, individuel ou groupal, repose sur la détention d'un savoir ou d'une compétence. C'est le cas des discours de transmission des connaissances (Beacco et Moirand 1995), des discours d'experts (Léglise et Garric 2012), et des discours médiatiques (Charaudeau 1997, Moirand 2007). Il s'agit donc prioritairement de genres de discours fortement ritualisés, qui remplissent tous ce que Bourdieu appelle les conditions "liturgiques" obligatoires pour qu'un discours d'autorité soit reconnu comme tel, à savoir "l'ensemble des prescriptions qui régissent la forme de la manifestation publique d'autorité » (Bourdieu 1982 : 111) et instaurent un cadre fortement contraint à l'intérieur duquel se déroule l'activité discursive.

Plusieurs travaux en sciences du langage et, notamment, en AD, interrogent les formes linguistiques et discursives que prennent les savoirs et les représentations collectives et partagées et leur rôle dans la construction de l'évidence et, par là, de l'autorité discursive (Guilbert 2015, Koren 2004). L'évidence permettrait en effet d'asseoir des propos sur des sources non explicitées, dont on ne connaît pas l'origine et qui semblent aller d'elles-mêmes.

Que ce soit sous la dénomination de doxa (Amossy 2002), de sens commun (Sarfati 1996, Longhi 2011), de stéréotype (Amossy 1991) ou de celles épistémologiquement plus situées de présupposition (Anscombre et Ducrot 1983), de compétence encyclopédique (Kerbrat-Orecchioni 1986) ou de prédiscours (Paveau 2006), ces recherches portent sur une grande variété d'unités linguistiques, discursives et discursivo-cognitives faisant l'objet d'un certain figement (Krieg-Planque 2015) qui inscrit en discours une autorité "sociale ». Celle-ci s'impose, de manière implicite, et tire sa force du déjà-là, de son effet de préconstruit; elle vise à s'imposer "naturellement", à faire consensus, en évitant tout type de clivage ou d'éclat (Monte et Oger 2015), et cela du moins dans des corpus homogènes du point de vue énonciatif.

Les modalités énonciatives qui permettent de construire un discours d'autorité et/ou de faire appel à une autorité légitime dans son propre discours ont fait également l'objet de recherches de la part d'analystes du discours. Les chercheurs ont montré le fonctionnement d'un large éventail de stratégies énonciatives de construction de l'autorité à travers la convocation d'autres voix en discours. Cela est bien visible dans les postures de co-, sous-, et sur-énonciation (Rabatel 2004) et de particitation (Maingueneau 2004) qui permettent de saisir les procédés à travers lesquels les énonciateurs expriment des points de vue surplombants ou ceux grâce auxquels ils inscrivent en discours un point de vue commun et partagé. D'un côté, ces stratégies rendent compte de l'importance des phénomènes d'hétérogénéité énonciative dans la construction discursive de postures d'autorité et, de l'autre, elles permettent d'insister sur l'importance des postures et des positionnements énonciatifs qui contribuent de 
manière massive à la création de l'ethos des locuteurs, comme dans le cas des discours d'expert (Garric et Léglise, 2012).

Si la question de l'autorité apparaît comme transversale à plusieurs approches en $\mathrm{AD}$, elle n'a fait que très rarement l'objet d'études systématiques. Deux numéros de Mots. Les langages du politique (Pineira et Périès 1995, Monte et Oger 2015) font figure d'exception et ont permis de montrer que les positions de Bourdieu, lorsqu'il affirme que " l'autorité vient au langage du dehors » (Bourdieu 1975 : 184), sont au moins à nuancer.

En effet, les analystes du discours ont mis au jour les procédés discursifs aptes à créer des discours d'autorité et, notamment, ont montré le rôle fondamental de la construction discursive de l'autorité via les deux dimensions principales de la légitimité et de la crédibilité à plusieurs niveaux de la structuration des discours. La prise en compte de ces dimensions suffit lorsque l'on analyse des corpus sinon clos, du moins homogènes du point de vue de la source énonciative, des lieux ou des dispositifs de communication. La prédilection pour des corpus «de groupe», de préférence des discours « autorisés » à partir de cadres institutionnels, médiatiques ou professionnels, a montré jusqu'à quel point la construction de l'autorité repose sur des opérations discursives de légitimation et de crédibilisation fondées sur des rituels et sur du déjàdit, à savoir sur le partage tacite de savoirs et de connaissances constituant le soubassement prédiscursif de tout discours marqué du sceau de l'autorité et garantissant ce qu'Oger (2013) a si bien appelé « surcroît de crédibilité ». Ce discours évite les clivages, prévient la contestation et se présente comme légitime et crédible à la fois à l'intérieur de communautés de locuteurs supposés partager un ensemble de pratiques, de valeurs et de normes, voire de prédiscours (Paveau 2006) qui garantissent une certaine homogénéité dans la reconnaissance de ce qui fait autorité dans un contexte donné.

Or, que se passe-t-il quand un discours tenu par une source énonciative ou un groupe d'énonciateurs homogène entre dans des « cercles de conversation » différents, comme les discours produits et partagés dans les dispositifs du web 2.0 dans des lieux différents de leur «lieu » d'énonciation d'origine et souvent non souhaités par ceux qui les ont produits? Quels défis épistémologiques, théoriques et méthodologiques se posent aux analystes pour observer la circulation de l'autorité dans des corpus «qui dérangent " (Courtine 1982 : 13), qui supposent, sans doute, un renouvellement au moins partiel des outils d'analyse (Paveau 2017, Marcoccia 2016) et un changement, du moins apparent, des critères de légitimation et de crédibilisation des discours, avec l'introduction de notions comme celles de popularité et de réputation?

\section{Autorité et argumentation : entre ethos et argument d'autorité}

En rhétorique et argumentation, la notion d'autorité fait l'objet d'une théorisation plus systématique, comme le montrent les nombreux travaux portant sur l'argument d'autorité (Plantin 2016, Angenot 2014) et sur une forme particulière d'appel à l'autorité, où elle est "auto-attribuée, incarnée et manifestée dans le discours de l'orateur » (Plantin 2016 : 107), c'est-à-dire l'ethos (Amossy 2014, 2010, 2010 [2000], 1999, Danblon 2006), qui paraissent d'intérêt majeur pour une problématisation discursive de la question de l'autorité.

21 L'ethos, à savoir l'image que l'orateur projette de soi dans son discours apparaît comme un composant fondamental de la dimension persuasive de tout discours en ce qu'il tient 
à la légitimité et la crédibilité de l'orateur (Amossy 2014, 2010). Ce sont justement ces deux dimensions qui sont à la base du fonctionnement de l'autorité rhétorique selon Danblon (2006). En effet, pour qu'il y ait autorité, d'un côté, il faut bien que l'auditoire reconnaisse l'orateur comme autorisé sur la base de plusieurs critères, qui tiennent à la place et au statut social occupés par l'orateur dans un contexte donné ; d'un autre côté, l'orateur se doit de construire en discours une image charismatique de soi-même, de se présenter comme digne de confiance. Pour ce faire, il doit éventuellement retravailler en discours les représentations de sa personne circulant dans l'espace social avant sa prise de parole, peut-être aussi dans le but de réparer son image ou de crédibiliser ses dires face à des opposants critiques qui ne reconnaissent pas son autorité comme allant de soi. Amossy (2010) conjugue heureusement différentes traditions philosophiques et rhétoriques en proposant une élaboration de la notion d'ethos qui reposerait sur le travail discursif (ethos discursif) que l'orateur opère à partir d'une dimension préalable à la mise en discours (ethos prédiscursif).

En appui à la dimension éthotique, le locuteur peut marquer son discours du sceau de l'autorité en recourant à l'argument d'autorité. Cela arrive lorsqu'il «donne pour argument en faveur d'une affirmation le fait qu'elle ait été énoncée par un locuteur particulier autorisé, sur lequel il s'appuie ou derrière lequel il se réfugie » (Plantin 1996 : 88). Les soupçons (Plantin 2016, Angenot 2014, Doury et Lerger 2006) dont ce type d'argument font l'objet dans la tradition rhétorique depuis l'Antiquité dérivent, selon Angenot, de deux visions différentes de l'autorité qui lient celle-ci tantôt à «la crédibilité, raisonnablement fondée c'est-à-dire argumentable, attribuée à une source compétente ", tantôt au "respect dû à une personne revêtue d'un pouvoir institué légitime» (2014: 11). Quel que soit le jugement que les spécialistes portent sur cet argument dans une optique normative, l'accord se situe au niveau de sa subordination à la preuve directe, qui lui est toujours préférable.

Comme le remarque Angenot, le recours à l'argument d'autorité subsume la question du crédit ou mieux de la confiance à accorder à l'autorité convoquée, cette confiance étant une «notion floue, difficile à circonscrire [...] mais élément essentiel et exigence constante de la vie en société » (2014:19).

La confiance dans le discours d'autrui est au cœur des mécanismes d'acquisition des connaissances dans les sociétés contemporaines, comme le soutiennent aussi les épistémologues (Origgi 2008), et cela contribue à déplacer la question de l'autorité vers l'identification des conditions nécessaires pour que les individus soient disposés à faire confiance au discours d'autrui, donc à reconnaître son autorité, tout en sachant que ces conditions varient en fonction des normes, des valeurs et des croyances propres à des cultures et des groupes sociaux plus ou moins étendus.

\section{Web 2.0 et enjeux d'autorité}

\section{Le Web 2.0 entre représentations et discours scientifiques et institutionnels}

La dénomination controversée de Web 2.0 a été proposée pour la première fois par Tim O'Reilly à la Web 2.0 conference d'O'Reilly Media à la fin de 2004 : elle désigne une " deuxième naissance du web [qui] s'est produite grâce à des interfaces qui permettent aux utilisateurs non seulement de consulter des sites, mais aussi de communiquer et 
d'échanger entre eux. Bref, un web beaucoup plus interactif et participatif » (Cardon 2019 : 152). Si dans l'usage ordinaire cette étiquette réfère surtout aux réseaux sociaux numériques (RSN), à savoir

sites as web-based services that allow individuals to (1) construct a public or semipublic profile within a bounded system, (2) articulate a list of other users with whom they share a connection, and (3) view and traverse their list of connections and those made by others within the system. (Boyd and Ellison, 2007: 210),

elle comprend également les forums, les blogs, les wikis et les dispositifs en ligne des journaux qui présentent de plus en plus d'outils de partage sur d'autres plateformes interconnectées dans leur macrostructure. L'accent y est donc mis sur les interactions entre usagers et sur une participation plus active de la part des internautes dans la création de contenus et dans le partage d'informations.

Cela dit, si dans les années 2000, au début du succès et de la diffusion de ces outils, l'on insistait sur la possibilité de partage, sur la plus grande facilité d'accès aux informations, sur une plus grande participation démocratique de la part de tous les citoyens aux questions politiques et sociales sur le ton enthousiaste de l'éloge, plus récemment, le web 2.0 fait l'objet d'un nombre croissant de discours sceptiques, surtout dans les médias - «Comment Emmanuel Macron veut "responsabiliser" les réseaux sociaux ", Le Monde, le 10 mai 2019, "Pour Castex, l'anonymat sur les réseaux sociaux rappelle le "régime de Vichy" ", Le Figaro, le 16/07/2020, "Réseaux sociaux : comment réguler sans (toujours) censurer?", Libération, le 22/01/2018.

Les RSN alimenteraient donc l'illusion d'un égalitarisme épistémique qui débouche volontiers sur un anarchisme épistémique où tout un chacun se sent autorisé à débattre autour de n'importe quel sujet, à prendre la parole en public et à affirmer ses positions plus ou moins justifiées sur la base de données objectives ou de compétences spécialisées (Origgi 2015). Bref - et il est désormais banal de le dire - l'autorité serait bien mise à mal dans ce genre de dispositifs. Le Web 2.0 n'aurait qu'accéléré une crise de l'autorité déjà entamée au cours du $20^{\mathrm{e}}$ siècle.

Ce point de vue serait bien sûr acceptable in toto si l'on considère que l'autorité renvoie aux seuls organes de pouvoir institutionnel, et si l'on oublie ses dimensions sociale, relationnelle et discursive, qui déterminent pourtant, la confiance octroyée par les citoyens aux institutions :

L'autorité des acteurs centraux n'est plus automatique, acquise et non négociable ; elle doit s'obtenir dans un monde ouvert, irrespectueux, bruyant, et parfois si désordonné que beaucoup y voient un immense bazar incontrôlable et dangereux. Les mondes numériques n'ont pourtant rien d'un capharnaüm. Ils proposent d'autres formes d'articulation entre citoyens et représentants, d'autres manières d'adresser des signaux aux médias, d'autres façons de se mobiliser et de faire de la politique (Cardon 2019 : 218).

Le Web 2.0 aurait donc entraîné un changement dans les processus de légitimation des discours institutionnels, tout comme de ceux émanant d'autres dispositifs communicationnels, de par la structure du moins apparemment horizontale qui le caractérise, et qui semble favoriser des phénomènes variés de négociation des savoirs et de contestation des institutions, comme le montre bien la formation en ligne de mouvements contestataires collectifs (les gilets jaunes, certains mouvements féministes, LGBTi, etc.). Dans la sphère médiatique, cela est très bien montré par l'analyse des dispositifs «commentaires » des journaux en ligne, où Calabrese (2014) constate le changement de statut des lecteurs en ligne dans la production et la 
circulation de l'information médiatique. Ceux-ci peuvent en effet réagir, rectifier, modifier les informations contenues dans les articles publiés par les journalistes et jouent par là un rôle plus actif, qui modifie les relations entre discours premier, professionnel, expert et discours second, « ordinaire », « profane ».

Sociologues, linguistes et spécialistes en sciences de l'information et de la communication n'ont pas manqué de remarquer la forte tendance à la polarisation dans les débats en ligne et par là l'exacerbation de la dimension polémique des débats en ligne (Mercier 2015, Amossy et Burger 2011), entrainant volontiers des manifestations de violence verbale (Monnier et al. 2021, Romain et Fracchiolla 2006). La polarisation des opinions est directement liée à la question de l'autorité. Elle constitue un terrain fertile pour la circulation de fausses nouvelles à l'intérieur des groupes qui se constituent dans les RSN (Smyrnaios et Marty 2017, Figeac et al. 2019), où les membres font souvent circuler des informations dont la légitimité de la source énonciative n'est pas toujours vérifiée. La diffusion des fausses nouvelles est par ailleurs favorisée par la pratique des trolls (Howard et al. 2018) si répandue dans les RSN. La construction de l'autorité tient alors davantage à des procédés directement observables dans la matérialité discursive, c'est-à-dire à des (re)configurations situées, qu'aux rôles et statuts plus ou moins légitimes des locuteurs qui en sont à l'origine. Sans doute, ces phénomènes alimentent et justifient du moins partiellement l'idée que dans les débats sur les plateformes du Web 2.0, aucune place ne semble faite à l'argumentation raisonnée :

une vaste mêlée hargneuse et haineuse, un champ de bataille où tous les coups de la rhétorique éristique sont permis, où tous les débats sans exception carburent à l'outrancier, tournent à l'invective, à la reductio ad Hitlerum ou ad Stalinam, au procès en règle, et notamment au procès d'intention instruit à la façon d'un réquisitoire vychinskyen, à la menace explicite très souvent, à la volonté de censurer, de réduire au silence (Angenot $2014: 382$ ).

Ce sont le désordre et le manque de règles qui domineraient dans le Web 2.0 et qui justifient, aux yeux des gouvernements, une intervention au niveau des politiques de réglementation : le contrôle des partages et de la diffusion de propos haineux, racistes, de contenus illégaux, de fausses nouvelles apparaît dès lors comme un enjeu majeur pour la plupart des démocraties contemporaines. Indépendamment des solutions préconisées par chaque autorité politique, le rôle des plateformes dans la lutte contre les dérives des contenus circulant dans le Web 2.0 est considéré comme insuffisant, tout comme la structure algorithmique sur laquelle il est fondé qui, comme on le verra, ne permet d'intervenir que de manière très faible pour limiter ces phénomènes.

\section{Web 2.0, réputation et autorité : quels enjeux pour l'analyse du discours?}

Pour appréhender la question de l'autorité et, donc, des mécanismes de confiance dans les discours du web 2.0 en $\mathrm{AD}$, il est nécessaire de comprendre les logiques et les mécanismes régissant l'écosystème dans lequel les discours sont produits et qui, en même temps, en permettent la construction et la circulation.

Les dispositifs du Web 2.0 ont mis en place des actions qui visent à réduire l'impact des phénomènes de distorsion dans la circulation des contenus en ligne. A côté des mesures "participatives» explicites, qui présupposent une participation active de la part des internautes aussi bien dans le respect des normes que dans la dénonciation de contenus 
illicites, c'est la structure implicite, sous-jacente, du Web 2.0, reposant sur certaines logiques algorithmiques, qui permet d'expliquer de nombreux phénomènes de (dé)construction de l'autorité.

Les algorithmes régissant le fonctionnement du Web 2.0 permettent de renouveler le paradigme notionnel autour de la notion d'autorité tel qu'il s'est dessiné sous la plume des philosophes du $20^{e}$ siècle qui se sont penchés sur cette notion. Les logiques algorithmiques invitent à aborder la notion d'autorité non plus seulement en relation avec des notions telles que pouvoir, norme, pertinence, expertise, charisme, etc., qui constituent le paradigme notionnel traditionnel, mais en la faisant basculer vers un autre paradigme de notions voisines: celles de visibilité, popularité, réputation, confiance, et influence comme l'ont montré des sociologues (Cardon 2019, Candel et Pergia 2017, Alloing 2014), des philosophes (Origgi 2008, 2015) et des spécialistes de l'information et de la communication (Broudoux 2007, Le Deuff 2007). Et notamment, la nécessité d'intégrer les paramètres technologiques dans l'analyse de la constitution de l'autorité dans les réseaux sociaux est témoignée par de récentes études en sciences de l'information et de la communication (Broudoux et Ihadjadene 2020). Ces études montrent tout l'intérêt de traiter de cette question à partir aussi du point de vue de l'AD, via l'identification et l'analyse précise des procédés discursifs révélant la construction et la circulation de l'autorité dans les médias sociaux.

Dans le web 2.0, la notion d'autorité s'enrichit d'une dimension technique. Pour faire autorité, il ne suffit pas d'être populaires, à savoir de recevoir un grand nombre de clics ou de likes, il faut aussi construire progressivement et dans le temps sa propre réputation. La notion d'autorité est en effet utilisée par les concepteurs des dispositifs pour nommer une série d'algorithmes permettant de dépasser des logiques purement quantitatives d'indexation et de trouvabilité des informations, grâce à la prise en compte du paramètre des liens hypertextuels entre les sites (qui pointe vers qui ?). Du point de vue technique, la mesure de l'autorité d'une page web serait ainsi déterminée par la présence sur d'autres sites de liens hypertextuels qui pointent vers la page ellemême. Ces algorithmes introduisent donc un indice de confiance extrait des pratiques hypertextuelles des usagers et permettent d'augmenter la réputation des résultats considérés comme plus pertinents.

Ce système entraîne un certain renouvellement des formes d'autorité par rapport aux types désormais traditionnels identifiés par Weber (traditionnelle; rationnelle et légale; charismatique), ces formes se fondant sur des pratiques intersubjectives de construction des connaissances et d'acquisition des savoirs permettant d'exercer une certaine influence, comme l'illustre bien la notion de réputation : «La réputation est [...] un phénomène d'intelligence collective : c'est le savoir des autres qui nous informe et c'est la façon dont l'autorité de ce savoir est construite qui nous donne confiance pour l'acquérir par le biais d'autrui » (Origgi 2015 : 194).

Cette notion se concrétise dans l'élaboration d'algorithmes qui modifient les logiques de circulation des discours dans le web social et qui établissent des hiérarchies d'informations sans que les internautes en soient toujours conscients sur la base de leurs intérêts et de ceux de leurs réseaux (Cardon 2010). Loin d'être stables et homogènes, ces hiérarchies se caractérisent par leur caractère dynamique. Si en effet, elles sont à la base de l'effet «bulle de filtre ", qui favorise l'exposition des internautes à des informations qui reflètent déjà leurs opinions, le plus souvent, ces bulles sont percées. La hiérarchie des informations n'empêche pas une circulation des discours qui 
est transversale à différents groupes sociaux, suivant les degrés de visibilité permis par les dispositifs et par les usagers à la fois. La rencontre de discours de groupes avec des standards épistémiques différents, des normes et des « rituels » variés, entraînerait une multiplication des instances de légitimation médiatiques, culturelles, institutionnelles ainsi qu'un brouillage des pratiques de légitimation et de crédibilisation des discours en fonction de la réduction de l'écart entre les deux types de confiance identifiées par Angenot (2014: 19) : l'horizontale, réciproque, qui se développe entre pairs et la verticale, qu'on octroie à une autorité supérieure, considérée comme plus légitime et crédible pour juger de quelque chose.

Dans cet état des choses, s'il est vrai que la déférence à certaines figures dépend de la structure des relations à l'intérieur du réseau (Origgi 2015), toute approche discursive de l'autorité dans le Web 2.0 se doit de prendre en compte les effets des affordances numériques des dispositifs (Ghliss et al. 2019) sous-jacentes à la production et à la circulation des discours et par là adopter des approches écologiques du discours qui tiennent en compte que « les discours numériques natifs ne sont pas d'ordre purement langagier» et "que les déterminations techniques co-construisent les formes technolangagières » (Paveau $2017:$ 11).

Ces affordances donnent ainsi la possibilité d'observer la stratification de la construction de l'autorité en discours et l'essor éventuel de conflits d'autorité surgissant au fil des échanges. C'est du moins l'hypothèse que les contributions de ce numéro permettront de valider à partir de la réponse aux questions suivantes :

- Peut-on identifier des manifestations de résistance ou de protestation face aux normes, à l'exercice du pouvoir, au discours autoritaire et/ou d'autorité ?

- Dans quelle mesure le non-partage des mêmes autorités par les locuteurs est à la base de la dimension conflictuelle déclenchant des polémiques (Amossy 2014b) qui circulent dans l'espace discursif public en ligne?

- Quelles modalités discursives et argumentatives plus ou moins conflictuelles sont utilisées par les locuteurs pour réfuter, délégitimer, ou au contraire, légitimer un discours d'autorité? Quels observables technodiscursifs doit-on prendre en compte pour cerner ces phénomènes?

\section{Présentation du dossier}

$41 \mathrm{Si}$ les contributions ici réunies partent d'approches écologiques des discours numériques, qui prennent toutes en compte la nature située des productions discursives dans le Web 2.0, elles permettent d'explorer la question de l'autorité dans le Web 2.0 à partir d'un large éventail de plateformes (forums, espace "commentaires » des journaux en ligne, Facebook, Twitter, Youtube, Instagram) et de cas de figure (groupes d'intérêt, pages et profils d'institutions, de locuteurs ordinaires, d'influenceurs, vidéos de journaux télévisés, etc.), par rapport à plusieurs discours «d'autorité " (médiatiques, politiques, scientifiques, médicaux, mais aussi ceux typiques du Web 2.0, comme les discours des influenceurs) et à travers la prise en compte des modalités discursives de (dé)construction et de circulation de l'autorité propres à chaque plateforme.

42 Les trois premières contributions interrogent les procédés de contestation de l'autorité des médias d'information traditionnels et des politiques. Et notamment Molinari et Bernard-Barbeau partent de la polémique autour de la formule «bonjour/hi » qui s'est 
déclenchée au sein de l'Assemblée nationale du Québec en 2017 pour montrer les différents moyens (techno)discursifs utilisés dans les médias traditionnels (presse) et dans le Web 2.0 (espace «commentaires" des journaux en ligne, Twitter et Facebook) dans le but de déconstruire l'autorité des politiques.

L'autorité politique est aussi au centre des deux contributions qui suivent. Gern analyse les réactions des internautes sur Youtube au discours d'Emanuel Macron en 2018, sur les liens entre l'Église et l'État, alors que Sadoun analyse celles que les twitteurs publient à la suite de l'allocution du Nouvel an de 2018 du président. Dans les deux cas, l'autorité présidentielle est confrontée aux technodiscours des internautes qui l'affaiblissent, voire la délégitiment, à partir d'un ample éventail de stratégies argumentatives et technodiscursives propres aux deux plateformes. Ces analyses rendent bien compte des enjeux discursifs et argumentatifs permettant la reconfiguration de la relation d'autorité entre citoyens-internautes et figures "traditionnelles» de l'autorité politique, institutionnelle, scientifique etc. qui sous-tend et régit l'organisation de nos sociétés libérales-démocratiques. Et notamment, ces études confirment l'hypothèse selon laquelle la rencontre de discours issus de locuteurs fort hétérogènes en termes de normes, valeurs et standards épistémiques favorise l'essor d'un espace polémique structuré par la remise en cause de l'autorité de ces figures et de leur légitimité.

Les trois dernières contributions mettent davantage l'accent sur les mécanismes de construction de nouvelles formes de discours d'autorité typiques du Web 2.0 tout en explorant des domaines fort différents comme celui techno-scientifique (Vicari), celui promotionnel de la pratique de l'unboxing (Attruia) et, enfin, le domaine médical (Charbonneaux et Berthelot).

45 Vicari analyse la polémique autour de la vape à partir des échanges des internautes et des profils d'institutions nationales et internationales dans différentes plateformes (espace « commentaires des journaux en ligne, Facebook, Twitter, Youtube) ainsi que la construction du discours des influenceurs de la vape sur Instagram, ceux-ci ayant contribué de manière massive au succès de cet outil de sevrage du tabac auprès d'un public jeune. Les discours des influenceurs font notamment l'objet d'une analyse fort détaillée dans la contribution d'Attruia. L'auteur s'attache à décrire comment les jeunes influenceurs construisent leur autorité dans les vidéos Youtube qui les saisissent en train de déballer des jouets. Dans la dernière contribution, Charbonneaux et Berthelot interrogent les processus d'autorisation qui se développent le long d'échanges sur des forums d'entraide médicale et notamment la fonction d'autorité jouée par la figure du " parent expert ». Les dynamiques des échanges montrent que loin de toute tentative de destitution de l'autorité médicale, le parent expert se situe plutôt dans une démarche de complémentarité avec la parole savante, dans le but d'exprimer un «jamais dit» issu de sa position de témoin. C'est une forme d'autorité davantage " collective ", fondée sur un modèle participatif et sur le partage avec les usagers de normes et de pratiques communes, qui se dessine dans ces discours.

Ces études montrent jusqu'à quel point réputation, autorité et influence sont étroitement liées dans ces espaces. Les environnements du Web 2.0, loin de se caractériser uniquement par la circulation dérèglée des informations et par la simple expression de réactions émotives, favorisent la mise en place de rituels discursifs fondés sur des procédés argumentatifs et discursifs plurisémiotiques, où dimensions technologique et langagière sont inséparables. Ces procédés permettent la construction de relations horizontales de confiance, marquées par une exigence accrue de proximité 
entre figures d'autorité et internautes. Bien que sans doute plus éphémères et mouvantes, ces relations témoignent d'un déplacement des critères de légitimation et de crédibilisation des discours dans les dynamiques intersubjectives des échanges. Elles montrent finalement tout l'intérêt d'une problématisation discursive des mécanismes de construction et de circulation de l'autorité dans le Web 2.0, qu'il faut appréhender en tant que phénomènes discursifs situés, à partir de la matérialité technodiscursive qui ressort d'études de cas de figure circonscrits.

\section{BIBLIOGRAPHY}

Adorno Theodor, W. \& Else Frenkel-Brunswik, Daniel Levinson, Nevitt Sanford. 1950. The Authoritarian Personality (New York: Harper \& Brothers)

Alloing, Camille. 2014. « Vers une approche instrumentale de l'identité numérique : les attributs identitaires comme structuration de l'environnement informationnel ? », Pinte, Jean-Paul (éd.). Enseignement, préservation et diffusion des identités numériques (Paris : Hermès Lavoisier), 39-68

Althusser, Louis. 2006 [1970]. « Idéologie et appareils idéologiques d'État. Sur la reproduction des conditions de production », Penser Louis Althusser (Pantin : Le temps des cerises)

Amossy, Ruth. 2014. "L'ethos et ses doubles contemporains. Perspectives disciplinaires ", Langage et société 149.3, 13-30 [En ligne] https://www.cairn.info/revue-langage-et-societe-2014-3page-13.htm (consulté le 28/01/2021)

Amossy, Ruth. 2014b. Apologie de la polémique (Paris : PUF)

Amossy, Ruth \& Marcel Burger (éds). 2011. « Introduction : la polémique médiatisée », Semen 31, « Polémiques médiatiques et journalistiques », 7-24

Amossy, Ruth. 2010. La présentation de soi. Ethos et identité verbale (Paris : PUF)

Amossy, Ruth. 2002. «Introduction to the Study of Doxa », Poetics Today 23.3, 369-394

Amossy, Ruth (éd.). 1999. Images de soi dans le discours. La construction de l'ethos (Lausanne : Delachaux et Niestlé)

Amossy, Ruth. 1991. Les idées reçues, Sémiologie du stéréotype (Paris : Nathan)

Angenot, Marc (2014), Rhétorique de la confiance et de l'autorité (Montréal : Discours social)

Anscombre, Jean-Claude \& Oswald Ducrot. 1983. L'argumentation dans la langue (Bruxelles : Mardaga)

Arendt, Hannah. 2004 [1955]. « Qu'est-ce que l'autorité ? », La crise de la culture (Paris : Gallimard), 138-158

Authier-Revuz, Jacqueline. 1995. Ces mots qui ne vont pas de soi. Boucles réflexives et non-coïncidences du dire, 2 vol. (Paris : Larousse)

Beacco, Jean-Claude. 1992. « Les genres textuels dans l'analyse du discours : écriture légitime et communautés translangagières », Langages 105, 8-27 
Beacco, Jean-Claude \& Sophie Moirand. 1995. « Autour des discours de transmission des connaissances ", Langages 117, 32-53

Bochenski, Józef Maria. 1974. Was ist Autorität? Einführung in die Logik der Autorität (Freiburg: Herder)

Bourdieu, Pierre. 1982. Ce que parler veut dire. L'économie des échanges linguistiques (Paris : Fayard)

Bourdieu, Pierre. 1977. « L'économie des échanges linguistiques », Langue française 34, 17-34

Bourdieu, Pierre. 1975. «Le langage autorisé. Notes sur les conditions sociales des discours rituels ", Actes de la Recherche en Sciences Sociales 5-6, 183-190

Boyd, Danah \& Nicole B. Ellison. 2007. « Social Network Sites: Definition, History, and Scholarship », Journal of Computer-mediated communication 13.1, 210-230

Broudoux, Évelyne \& Madjid Ihadjadene. 2020. « Introduction », Études de communication 55, « La constitution des autorités numériques dans la production et la circulation de l'information », $7-14$

Broudoux, Evelyne. 2007. « Construction de l'autorité informationnelle sur le web », SKARE Roswitha, Niels Windfeld Lund \& Andreas Vårheim (éds), A Document (Re)turn: Contributions From a Research Field in Transition (Frankfurt am Main: P. Lang), 1-11

Calabrese, Laura. 2014. « Rectifier le discours d'information médiatique : quelle légitimité pour le discours profane dans la presse d'information en ligne? ", Les Carnets du Cediscor 12. En ligne : http://cediscor.revues.org/916 [Page consultée le 28/01/2021].

Candel, Étienne \& Pergia, Gkouskou-Giannakou. 2017. « Autorité et pratiques de légitimation en ligne », Quaderni 93, « Autorité et pratiques de légitimation en ligne », 5- 11.

Cardon, Dominique. 2010. La Démocratie Internet. Promesses et limites, Paris, Seuil/La République des idées.

Certeau, Michel (de). 1974. La culture au pluriel (Paris : Union générale d'éditions).

Charaudeau, Patrick. 2017. Le débat public. Entre controverse et polémique. Enjeu de vérité, enjeu de pouvoir (Limoges : Lambert-Lucas).

Charaudeau, Patrick. 2005. Le discours politique. Les masques du pouvoir (Paris : Vuibert).

Charaudeau, Patrick. 1997. Le discours d'information médiatique. La construction du miroir social (Paris : Nathan-INA).

Couleau, Christèle, Oriane, Deseilligny et Pascale Hellégouarc'h (éds.). 2016. « Ethos numériques », Itinéraires, 2015-3. [En ligne] https://doi.org/10.4000/itineraires.2953 (consulté le 28/01/2021)

Courtine, Jacques. 1982. «L'instituteur et le militant (Contribution à l'histoire de l'analyse du discours en France) ", Archives et documents de la Société d'histoire et d'épistémologie des sciences du langage 2, 1-15

Danblon, Emmanuelle. 2006. « La construction de l'autorité en rhétorique », Semen 21 [En ligne] http://journals.openedition.org/semen/1983 (consulté le 28/01/2021)

Doutreix, Marie-Noëlle \& Lionel Barbe. 2019. « Légitimer et disqualifier : les Fake News saisies comme opportunité de normalisation du champ journalistique », Études de communication 53, 49-66 
Doury, Marianne. 2008. «Ce n'est pas un argument !» Sur quelques aspects des théorisations spontanées de l'argumentation, Pratiques 139-140, 111-128

Doury, Marianne \& Céline Largier. 2006. « L'argument d'autorité dans une discussion en ligne : manifestations discursives et régime d'acceptabilité ", Actes $2 e$ Journées d'études internationales sur "L'argumentation et la rhétorique dans le domaine arabe » (L'argument d'autorité dans les textes et les cultures arabes et européennes. Pour un dialogue entre les traditions de recherche en argumentation), Tunis, 24-25 novembre 2006 (jamais paru ; disponible uniquement [En ligne] https:// ita.calameo.com/books/002834800201f19b1d95a (consulté le 28/01/2021)

Durkheim, Émile. 1925. L'Éducation morale (Paris : Alcan)

Figeac Julien, Tristan Salord, Guillaume Cabanac, Ophélie Fraisier, Pierre Ratinaud, Fanny Seffusatti \& Nikos Smyrnaios. 2019. « Facebook favorise-t-il la désinformation et la polarisation idéologique des opinions? ", Questions de communication 36, « Des données urbaines », 167-187

Foucault, Michel. 2009. Le courage de la vérité. Le gouvernement de soi et des autres II. Cours au collège de France 1984 (Paris : Gallimard/Seuil)

Foucault, Michel. 2008 [1969]. L'archéologie du savoir (Paris: Gallimard)

Foucault, Michel. 1975. Surveiller et punir. Naissance de la prison (Paris : Gallimard)

Garrau, Marie. 2018. Politiques de la vulnérabilité (Paris : CNRS éditions)

Ghliss, Yosra, François, Perea \& Catherine Ruchon (éds). 2019. «Introduction : Les affordances langagières, levier d'une réflexion postdualiste du discours numérique ? ", Corela 28 [En ligne] http://journals.openedition.org/corela/8282 (consulté le 28/01/2021)

Guilbert, Thierry. 2015. « Autorité et évidence discursives. Autovalidation dans les éditoriaux et chroniques du Point », Mots. Les langages du politique 107, 85-99

Guilbert, Thierry. 2014. « Les genres du discours dans l'articulation des approches qualitatives et quantitatives », Corela 15 [En ligne] https://doi.org/10.4000/corela.3577 (consulté le 28/01/2021)

Howard, Philippe, Bharath Ganesh, Dimitra Liotsiou, John Kelly, \& Camille Francois.2018. The IRA, social media and political polarization in the United States, 2012-2018 (Oxford, UK: Project on Computational Propaganda) [En ligne] https://comprop.oii.ox.ac.uk/wp-content/uploads/sites/ 93/2018/12/IRA-Report-2018.pdf (consulté le 28/01/2021)

Kerbrat-Orecchioni, Catherine. 1986. L'implicite (Paris : Colin)

Kojève, Alexandre. 2004. La notion de l'autorité (Paris : Gallimard)

Koren, Roselyne. 2004. «Argumentation, enjeux et pratique de l'“engagement neutre”. Le cas de l'écriture de presse », Semen 17 [En ligne] https://doi.org/10.4000/semen.2308 (consulté le 28/01/2021)

Krieg-Planque, Alice. 2015. « Construire et déconstruire l'autorité en discours. Le figement discursif et sa subversion », Mots. Les langages du politique 107, « Discours d'autorité : des discours sans éclat(s) ?», 115-132

Le Deuff, Olivier. 2007. « Autorité et pertinence vs popularité et influence : réseaux sociaux sur Internet et mutations institutionnelles » [En ligne] https://archivesic.ccsd.cnrs.fr/sic_00122603/ document (consulté le 28/01/2021)

Leclerc, Gérard. 1996. Histoire de l'autorité. L'assignation des énoncés culturels et la généalogie de la croyance (Paris : PUF) 
Léglise, Isabelle \& Nathalie Garric. 2012. « Analyser le discours d'expert et d'expertise », Léglise, Isabelle \& Nathalie Garric (éds). Discours d'experts et d'expertise (Berne : P. Lang), 1-16

Longhi, Julien. 2011. Visées discursives et dynamiques du sens commun (Paris : L'Harmattan)

Maingueneau, Dominique. 2012. «Que cherchent les analystes du discours? » Argumentation et Analyse du Discours 9 [En ligne] doi:https://doi.org/10.4000/aad.1354 (consulté le 28/01/2021)

Maingueneau, Dominique. 2010. «Le discours politique et son “environnement" », Mots. Les langages du politique $94,85-90$

Maingueneau, Dominique. 2005. «L'analyse du discours et ses frontières », Marges linguistiques 9, 64-75

Maingueneau, Dominique. 1993. « Analyse du discours et archive », Semen 8 [En ligne] doi:https:// doi.org/10.4000/semen.4069

Maingueneau, Dominique. 1992. «Le tour ethnolinguistique de l'analyse du discours ». Langages $105,114-125$

Maingueneau, Dominique \& Patrick Charaudeau. 2002. Dictionnaire d'analyse du discours (Paris: Seuil)

Maingueneau, Dominique \& Frédéric Cossutta. 1995. «L'analyse des discours constituants ", Langages 117, 112-125

Marcoccia, Michel. 2016. Analyser la communication numérique écrite (Paris : Colin).

Mercier, Arnaud. 2015. « Twitter, espace polémique, espace politique. L'exemple des tweetscampagne municipale en France (janvier-mars 2014) », Les Cahiers du numérique 4, 145-169

Milgram, Stanley. 1963. «Behavioral Study of obedience », The Journal of Abnormal and Social Psychology 67.4, 371-378

Moirand, Sophie. 2007. Les discours de la presse quotidienne. Observer, analyser, comprendre (Paris : PUF)

Monte, Michèle \& Claire Oger (éds). 2015. « Discours d'autorité : des discours sans éclat(s) ? ", Mots. Les langages du politique 107

Oger, Claire. 2019. « Formation dicursive » Publictionnaire. Dictionnaire encyclopédique et critique des publics [En ligne] http://publictionnaire.huma-num.fr/notice/formation-discursive (consulté le 28/01/2021)

Oger, Claire. 2013. Discours d'autorité, discours autorisés. Faire référence et dire l'institution, « inédit » du dossier d'habilitation à diriger des recherches en sciences de l'information et de la communication, soutenu le 2 décembre 2013 à l'Université Paris-Sorbonne (CELSA)

Oger, Claire. 2005. «L'analyse du discours institutionnel entre formations discursives et problématiques socio-anthropologiques », Langage et société 114, « Approches interdisciplinaires des pratiques langagières et discursives ", 113-128

Origgi, Gloria. 2015. La réputation. Qui dit quoi de qui (Paris : PUF)

Origgi, Gloria. 2008. Qu'est-ce que la confiance? (Paris : Vrin)

Origgi, Gloria. 2008b. « Trust, authority and epistemic responsibility », Theoria 61, 35-44

Paveau, Marie-Anne. 2017. L'Analyse du discours numérique. Dictionnaire des formes et des pratiques (Paris : Hermann) 
Paveau, Marie-Anne. 2006. Les prédiscours. Sens, mémoire, cognition (Paris : Presses de la Sorbonne Nouvelle)

Perelman, Chaïm, 1990, Éthique et droit (Bruxelles : Éditions de l'Université de Bruxelles)

Plantin Christian. 2016. Dictionnaire de l'argumentation. Une introduction aux études d'argumentation (Lyon : ENS Éditions)

Pineira, Carmen \& Gabriel Périès (éds). 1995. « Actes d'autorité. Discours autoritaires », Mots. Les langages du politique 43

Rabatel, Alain. 2004. «L'effacement énonciatif dans les discours rapportés et ses effets pragmatiques », Langages 154, 3-17

Rastier, François. 2001. Arts et sciences du texte (Paris : PUF)

Raz Joseph. 1979. The Authority of Law (Oxford: Clarendon Press)

Romain Christina \& Béatrice Fracchiolla. 2016. «Violence verbale et communication numérique écrite : la communication désincarnée en question », Cahiers de praxématique 66, " Emotions en contextes numériques » [En ligne] https://journals.openedition.org/praxematique/4263 [(consultée le 28/01/2021)

Sarfati, Georges-Elia. 1996. La sémantique : de l'énonciation au sens commun. Eléments pour une pragmatique topique, synthèse pour l'habilitation, Université de Paris 4 [En ligne] http:// www.revue-texto.net/Inedits/Sarfati/Sarfati_Semantique.html (consultée le 28/01/2021) Smyrnaios Nikos \& Emmanuel Marty. 2017. «Profession « nettoyeur du net ». De la modération des commentaires sur les sites d'information français », Réseaux 205, « Modèles économiques, usages et pluralisme de l'information en ligne », $57-90$

Vincent, Diane. 2013. «L'agression verbale comme mode d'acquisition d'un capital symbolique », Fracchiolla, Béatrice, Claudine Moïse, Christina Romain \& Nathalie Auger (éds). Violences verbales. Analyses, enjeux et perspectives (Rennes : P. U. de Rennes), 37-53

Weber, Max. 1922. « Die drei reinen Typen der legitimen Herrschaft », Preussische Jahrbücher 187, $1-12$

\section{AUTHOR}

\section{STEFANO VICARI}

Università di Genova 